\title{
Identificação LPV de Sistemas Não-Lineares via Ajuste Polinomial de Coeficientes
}

\author{
Itamar Pifano Silva* Roberto Ades** \\ Paulo César Pellanda ${ }^{* * *}$
Instituto Militar de Engenharia, Rio de Janeiro, RJ
*e-mail: itamarps@ime.eb.br
**e-mail: rades@ime.eb.br
*** e-mail: pcpellanda@ieee.org

\begin{abstract}
This work presents a method for the identification or tuning of LPV models with polynomial coefficients. The method is applicable to multivariable systems and also to approximate the behavior of possibly non-linear systems. The maximum pole modulus of the identified LPV model, for fixed values in the parameter trajectory, is also used for identification quality analysis. A second order mass-spring system and another fourth-order example are used to illustrate the application of this method.
\end{abstract}

Resumo: Este trabalho apresenta um método para a identificação ou ajuste de modelos LPV com coeficientes polinomiais. O método é aplicável a sistemas multivariáveis e também para a aproximação do comportamento de sistemas possivelmente não-lineares. O módulo máximo dos polos do modelo LPV identificado, para valores fixos na trajetória do parâmetro, também é utilizado para análise da qualidade da identificação. Para ilustrar a aplicação do método, são utilizados um sistema massa-mola de segunda ordem e outro exemplo de quarta ordem.
Keywords: System Identification; Nonlinear Systems; LPV Systems; Quasi-LPV; Polynomial Approximation.
Palavras-chaves: Identificação de Sistema; Sistemas Não-Lineares; Sistemas LPV; Quasi-LPV; Aproximação Polinomial.

\section{INTRODUÇÃO}

A área de identificação de sistemas lineares teve um desenvolvimento significativo a partir da década de 1970. No entanto, para o caso dos sistemas não-lineares, não se observou avanço similar restando muito a ser explorado. Os métodos de identificação de sistemas visam a obtenção de modelos a partir dos sinais medidos das entradas e saídas do sistema em estudo (Ljung, 1999). Basicamente, podem ser classificados em duas frentes, a identificação paramétrica e a não-paramétrica. $\mathrm{O}$ caso da identificação não-paramétrica envolve uma estrutura não determinada e, consequentemente, um número não determinado a priori de parâmetros. Quanto à estimação paramétrica, a estrutura a ser identificada encontra-se estabelecida previamente e um número determinado de parâmetros é ajustado. A identificação paramétrica em conjunto com a modelagem tem por objetivo aumentar a realidade do modelo e validá-lo por meio do ajuste de seus parâmetros. Normalmente, a metodologia de modelagem e as técnicas de identificação de sistema são empregadas separadamente. A utilização conjunta das abordagens tem por objetivo aumentar a precisão do modelo e uma técnica acaba servindo para validar a outra (Ljung and Glad, 2016).

* O presente trabalho foi realizado com apoio da Coordenação de Aperfeiçoamento de Pessoal de Nível Superior - Brasil (CAPES) Código de Financiamento 001.
Na realidade, a maioria dos sistemas dinâmicos existentes na prática envolvem dinâmicas não-lineares e, consequentemente, o processo de identificação voltado para sistemas lineares pode não ser tão útil para as aplicações de interesse. Essa limitação, somada à necessidade crescente de obtenção de modelos para a análise e controle de sistemas não-lineares, tem aumentado a demanda por métodos de identificação de sistemas não-lineares (Aguirre, 2015) (Lovera et al., 2013).

O modelo em espaço de estados é a forma matemática mais comum de representar um sistema não-linear:

$$
\begin{gathered}
\dot{x}=f(t, x, u), \\
y=g(t, x, u) .
\end{gathered}
$$

em que $x \in \mathbb{R}^{n}$ é o vetor de estados do sistema, $u \in \mathbb{R}^{q}$ é o vetor de entradas, $y \in \mathbb{R}^{l}$ é o vetor de saídas, $t$ é a variável tempo, $f$ e $g$ são as funções vetoriais não-lineares e a dimensão $n$ é a ordem do sistema.

Foi observado, em (Verdult, 2002), que a particular escolha das funções $f$ e $g$ para as equações em espaço de estado determina a estrutura de não linearidade do modelo. Claro que existem muitas possibilidades. Em geral, duas abordagens podem ser realizadas. A primeira delas é a escolha de uma estrutura simples para o modelo, de modo que se tenham certas propriedades desejáveis que facilitem a análise e que conduzam a métodos de identificação computacionais que exijam menor esforço. A principal desvanta- 
gem dessa estrutura é que frequentemente representa uma classe limitada de sistemas não-lineares, que não obrigatoriamente se ajusta bem aos sistemas práticos sob análise. A segunda opção, mais complexa, objetiva a seleção de estruturas de modelos que possam aproximar uma ampla classe de modelos não-lineares, mas com tratamento mais difícil. Exemplos de estruturas da primeira abordagem são os modelos lineares, bilineares, de Hammerstein e de Wiener. Já na segunda abordagem estão os modelos de redes neurais, de rede de função de base radial e os modelos fuzzy Takagi-Sugeno.

Em termos de controle, entre as abordagens propostas na literatura para sistemas não-lineares consta o clássico método de gain scheduling. Apesar de ser bastante empregado, este não garante a estabilidade e o desempenho fora dos pontos de operação considerados nos projetos lineares, especialmente nos casos em que o parâmetro varia rapidamente. O controle LPV (Linear Parameter Varying), com estabilidade local ou global e desempenho garantidos em grandes envelopes do domínio de operação de sistemas multivariáveis, tem se apresentado como uma alternativa real ao clássico método de gain scheduling. Nesse sentido, uma condição necessária para se realizar a síntese de controladores LPV é a transformação do modelo não-linear em um modelo LPV ou Quasi-LPV, que é o foco deste trabalho.

Neste artigo, foram adotadas algumas ideias apresentadas em (Bamieh and Giarré, 1999, 2002), tendo sido propostos os seguintes desenvolvimentos adicionais: extensão do método para sistemas multivariáveis; obtenção da solução a partir de um lote de dados armazenados ao invés da estimação pela forma recursiva; utilização de polinômios com graus independentes em cada coeficiente do modelo; e a possibilidade de realizar a defasagem temporal no modelo entre a saída corrente e a entrada mais recente, que corresponde ao aumento do grau relativo da função de transferência do modelo identificado. Adicionalmente, procurou-se explorar exemplos mais complexos que evidenciam problemas de estabilidade do modelo, não abordados nos trabalhos citados.

\section{SISTEMAS LPV / QUASI-LPV}

Em (Marcos and Balas, 2004), é apresentado o desenvolvimento de modelos LPV e Quasi-LPV utilizando três métodos: por linearização jacobiana, por transformação de estados e, pela substituição de funções. Também são mostradas suas vantagens e desvantagens pela aplicação em modelos de aeronaves de asa fixa para um dado envelope de voo.

O modelo LTV (Linear Time Varying) é um caso particular de sistema LPV em que as matrizes da dinâmica dependem do parâmetro variante $\theta(t)=t$.

Definição 1 - Modelo LPV (Becker, 1993), apud (Marcos and Balas, 2004): Dado um subconjunto compacto $\mathcal{P} \subset$ $\mathbb{R}^{d}, \mathcal{F}_{\mathcal{P}}$ representa o operador que mapeia $t \in \mathbb{R}^{+}$em um vetor $\theta(t) \in \mathcal{P}$, cujas componentes são funções contínuas por partes $\forall t \in \mathbb{R}^{+}$. Considere ainda as funções contínuas, $A: \mathbb{R}^{d} \rightarrow \mathbb{R}^{n \times n}, B: \mathbb{R}^{d} \rightarrow \mathbb{R}^{n \times q}, C: \mathbb{R}^{d} \rightarrow \mathbb{R}^{l \times n} \mathrm{e}$
$D: \mathbb{R}^{d} \rightarrow \mathbb{R}^{l \times q}$. Um modelo LPV de ordem $n$ é definido como:

$$
\left[\begin{array}{l}
\dot{x}(t) \\
y(t)
\end{array}\right]=\left[\begin{array}{ll}
A(\theta(t)) & B(\theta(t)) \\
C(\theta(t)) & D(\theta(t))
\end{array}\right]\left[\begin{array}{l}
x(t) \\
u(t)
\end{array}\right] .
$$

Um modelo Quasi-LPV é um modelo não-linear que se assemelha ao modelo LPV em (3). Nesse caso, o vetor $\theta(t)$ é composto por dois tipos de parâmetros variantes, os exógenos e os endógenos. Os parâmetros exógenos são variáveis externas que interferem no modelo e correspondem àquelas do modelo LPV, consideradas disponíveis por medição. Os parâmetros endógenos são funções de estados do sistema, que devem ser mensuráveis, uma vez que também interferem nas matrizes da dinâmica.

Definição 2 - Modelo Quasi-LPV (Marcos and Balas, 2004): Seja $\theta(t) \in \mathcal{P}$ tal que $\theta(t)=\left[\Omega(t)^{T} z(t)^{T}\right]^{T}$, onde $\Omega(t)$ corresponde ao vetor das variáveis exógenas, de forma similar ao sistema LPV em (3), e $z(t)$ corresponde ao vetor contendo alguns estados do sistema ou funções destes. O modelo Quasi-LPV pode ser representado por:

$$
\left[\begin{array}{c}
\dot{z}(t) \\
\dot{w}(t) \\
y(t)
\end{array}\right]=\left[\begin{array}{ccc}
A_{11}(\theta(t)) & A_{12}(\theta(t)) & B_{1}(\theta(t)) \\
A_{21}(\theta(t)) & A_{22}(\theta(t)) & B_{2}(\theta(t)) \\
C_{1}(\theta(t)) & C_{2}(\theta(t)) & D(\theta(t))
\end{array}\right]\left[\begin{array}{c}
z(t) \\
w(t) \\
u(t)
\end{array}\right],(4)
$$

em que o vetor de estados $x(t)=\left[z(t)^{T} w(t)^{T}\right]^{T}$, onde $w(t)$ corresponde aos demais estados do sistema, que não interferem nas matrizes do modelo.

Conforme (Rugh and Shamma, 2000), à título de exemplificação, considere a planta não-linear representada pelas seguintes equações:

$$
\begin{aligned}
& \dot{x}_{1}=\operatorname{sen}\left(x_{1}\right)+x_{2}, \\
& \dot{x}_{2}=x_{1} x_{2}+u .
\end{aligned}
$$

Uma representação Quasi-LPV desse modelo não-linear poderia ser:

$$
\dot{x}=\left[\begin{array}{cc}
\operatorname{sen}\left(x_{1}\right) / x_{1} & 1 \\
x_{2} & 0
\end{array}\right] x+\left[\begin{array}{l}
0 \\
1
\end{array}\right] u .
$$

Essa representação pode não ser adequada, a menos que $x_{1}$ e $x_{2}$ sejam mensuráveis. Nesse caso, $x_{1} \neq 0, \theta(t)=z(t)$, $x(t)=\left[\begin{array}{ll}x_{1} & x_{2}\end{array}\right]^{T}=z(t)$ e $w(t)=\Omega(t)=\varnothing$. Supondo que se tenha somente $x_{1}$ mensurável, uma representação mais adequada poderia ser:

$$
\dot{x}=\left[\begin{array}{cc}
\operatorname{sen}\left(x_{1}\right) / x_{1} & 1 \\
0 & x_{1}
\end{array}\right] x+\left[\begin{array}{l}
0 \\
1
\end{array}\right] u
$$

que permite reescrever a equação em uma forma em que a matriz de transição de estado somente possui dependência da variável $x_{1}$, ou seja, $z(t)=x_{1}$ e $w(t)=x_{2}$.

\section{MÉTODO PROPOSTO}

O problema aqui tratado consiste em ajustar um modelo LPV, de forma que a sua saída $\hat{y}$ se aproxime, segundo a norma quadrática, da saída $y$ do sistema físico não linear. A proposta é que o modelo seja discreto e esteja sob a forma de uma série temporal, cujos coeficientes 
possam ser polinômios dependentes do parâmetro variante $\theta=\theta(t)$. Para identificação do modelo, considera-se que o sistema sob estudo tenha sido previamente sensoriado, de forma que os dados temporais de suas entradas, saídas e do parâmetro variante, ainda que contínuos, tenham sido armazenados segundo um conveniente período de amostragem $T$.

Do mesmo modo que em (Bamieh and Giarré, 1999, 2002), adotou-se a classe de modelos LPV discretos no tempo, parametrizados da seguinte forma:

$$
\mathcal{A}(\delta, \theta) y(k)=\mathcal{B}(\delta, \theta) u(k),
$$

em que $\delta$ é o operador de atraso, $\delta^{n} y(k) \triangleq y(k-n)$ e

$$
\begin{aligned}
& \mathcal{A}(\delta, \theta) \triangleq 1+a_{1}(\theta) \delta+\cdots+a_{n}(\theta) \delta^{n}, \\
& \mathcal{B}(\delta, \theta) \triangleq b_{1}(\theta) \delta^{r}+b_{2}(\theta) \delta^{r+1}+\cdots+b_{m}(\theta) \delta^{r+m-1} .
\end{aligned}
$$

Além disso, considerou-se que o parâmetro variante $\theta$ é função do tempo discreto, $\theta \triangleq \theta(k T) \triangleq \theta_{k}, n$ representa o número de termos autorregressivos do sinal de saída e a ordem do modelo, $m$ é o número de termos defasado da entrada, $r$ é a defasagem entre a saída atual e a entrada mais recente considerada, e $m+r-1 \leq n$.

Assim, por (5) e (6), a estrutura do modelo identificado em forma de séries temporais é dada por:

$$
\begin{aligned}
& y_{k}=-a_{1} y_{k-1}-a_{2} y_{k-2}-\cdots-a_{n} y_{k-n}+ \\
& +b_{1} u_{k-r}+b_{2} u_{k-r-1}+\cdots+b_{m} u_{k-r-m+1},
\end{aligned}
$$

em que $y_{k} \triangleq y(k T)$ e $u_{k} \triangleq u(k T)$.

Considerou-se, ainda, que os coeficientes do modelo acima possuem uma dependência polinomial em relação ao parâmetro variante $\theta=\theta_{k}$. Assim, $\forall i \in\{1, \cdots, n\}$ e $N_{i} \in \mathbb{N}$ :

$$
a_{i}=a_{i}(\theta)=\alpha_{i, 0}+\alpha_{i, 1} \theta+\alpha_{i, 2} \theta^{2}+\cdots+\alpha_{i, N_{i}} \theta^{N_{i}} .
$$

Da mesma forma, para os coeficientes da variável de entrada $u, \forall j \in\{1, \cdots, m\}$ e $M_{j} \in \mathbb{N}$ :

$$
b_{j}=b_{j}(\theta)=\beta_{j, 0}+\beta_{j, 1} \theta+\beta_{j, 2} \theta^{2}+\cdots+\beta_{j, M_{j}} \theta^{M_{j}} .
$$

O parâmetro variante $\theta(t)$ é considerado mensurável, mas pode estar defasado da saída corrente, ou seja:

$$
y_{k}=f\left(\theta_{k-c}\right)
$$

em que $c \in\{0, \cdots, n\}$. Usualmente, adota-se $c=1$, ou seja, a determinação da saída atual depende do valor de $\theta$ no instante anterior.

Embora tenha sido adotado a estrutura em (7) na forma de série temporal, é importante observar que esta possui uma correspondência biunívoca com modelos em espaço de estado, bastando a utilização de uma realização canônica.

Tendo em vista os exemplos na seção seguinte, definem-se os seguintes vetores, contendo as ordens dos polinômios:

$$
\begin{aligned}
& \mathbf{N} \triangleq\left[\begin{array}{llll}
N_{1} & N_{2} & \cdots & N_{n}
\end{array}\right], \\
& \mathbf{M} \triangleq\left[\begin{array}{llll}
M_{1} & M_{2} & \cdots & M_{m}
\end{array}\right] .
\end{aligned}
$$

O número $\nu$ de parâmetros a serem identificados pode ser calculado por meio de:

$$
\nu=n+m+\sum_{i=1}^{n} N_{i}+\sum_{j=1}^{m} M_{j}
$$

O teorema a seguir mostra como calcular os coeficientes polinomiais a serem identificados, a partir da resolução de um sistema de equações lineares e dos dados de entrada, saída e do parâmetro variante medidos previamente no sistema físico.

Teorema 1 - Considere $u_{k}, y_{k}$ e $\theta_{k}, \operatorname{com} k \in\{1, \cdots, p\}$, as séries de dados medidos referentes, respectivamente, à entrada, à saída e ao parâmetro variante de um sistema a ser identificado. Os coeficientes polinomiais em (8) e (9) do modelo LPV podem ser determinados por meio do seguinte sistema de equações lineares:

$$
A X=\left[\begin{array}{ll}
A_{\alpha} & A_{\beta}
\end{array}\right]\left[\begin{array}{l}
X_{\alpha} \\
X_{\beta}
\end{array}\right]=B,
$$

em que:

$$
\begin{aligned}
& A_{\alpha}=\left[\begin{array}{cccc}
Y_{n-1, N_{1}} & Y_{n-2, N_{2}} & \cdots & Y_{0, N_{n}} \\
Y_{n, N_{1}} & Y_{n-1, N_{2}} & \cdots & Y_{1, N_{n}} \\
\vdots & \vdots & \ddots & \vdots \\
Y_{p-1, N_{1}} & Y_{p-2, N_{2}} & \cdots & Y_{p-n, N_{n}}
\end{array}\right] \\
& A_{\beta}=\left[\begin{array}{cccc}
U_{n-r, M_{1}} & U_{n-r-1, M_{2}} & \cdots & U_{n-r-m+1, M_{m}} \\
U_{n-r+1, M_{1}} & U_{n-r, M_{2}} & \cdots & U_{n-r-m+2, M_{m}} \\
\vdots & \vdots & \ddots & \vdots \\
U_{p-r, M_{1}} & U_{p-r-1, M_{2}} & \cdots & U_{p-r-m+1, M_{m}}
\end{array}\right] \text {, } \\
& X_{\alpha}=-\left[\begin{array}{lllllll}
\alpha_{1,0} & \alpha_{1,1} & \cdots & \alpha_{1, N_{1}} & \alpha_{2,0} & \cdots & \alpha_{n, N_{n}}
\end{array}\right]^{T}, \\
& X_{\beta}=\left[\begin{array}{lllllll}
\beta_{1,0} & \beta_{1,1} & \cdots & \beta_{1, M_{1}} & \beta_{2,0} & \cdots & \beta_{m, M_{m}}
\end{array}\right]^{T}, \\
& B=\left[\begin{array}{lllll}
y_{n} & y_{n+1} & y_{n+2} & \cdots & y_{p}
\end{array}\right]^{T} \in \mathbb{R}^{p-n+1}, \\
& Y_{k, p} \triangleq y_{k}\left[\begin{array}{lllll}
1 & \theta_{k-c} & \theta_{k-c}^{2} & \cdots & \theta_{k-c}^{p}
\end{array}\right] \in \mathbb{R}^{p+1}, \\
& U_{k, p} \triangleq u_{k}\left[\begin{array}{lllll}
1 & \theta_{k-c} & \theta_{k-c}^{2} & \cdots & \theta_{k-c}^{p}
\end{array}\right] \in \mathbb{R}^{p+1} .
\end{aligned}
$$

Demonstração : O sistema de equações lineares em (12) decorre diretamente de (7). Em um determinado instante de tempo $t=k T$, a equação (7) pode ser reescrita com o auxílio de (8) e (9) da seguinte forma:

$$
\begin{array}{r}
y_{k}=-\left(\alpha_{1,0}+\cdots+\alpha_{1, N_{1}} \theta^{N_{1}}\right) y_{k-1}-\cdots \\
-\left(\alpha_{n, 0}+\cdots+\alpha_{n, N_{n}} \theta^{N_{n}}\right) y_{k-n}+ \\
+\left(\beta_{1,0}+\cdots+\beta_{1, M_{1}} \theta^{M_{1}}\right) u_{k-r}+\cdots \\
+\left(\beta_{m, 0}+\cdots+\beta_{m, M_{m}} \theta^{M_{m}}\right) u_{k-r-m+1},
\end{array}
$$

que pode ser reescrito como:

$$
\begin{aligned}
& y_{k}=\left[\begin{array}{lllll}
Y_{k-1, N_{1}} & \cdots & Y_{k-n, N_{n}} & U_{k-r, M_{1}} & \cdots
\end{array}\right. \\
& \left.\cdots U_{k-r-m+1, M_{m}}\right]\left[\begin{array}{c}
X_{\alpha} \\
X_{\beta}
\end{array}\right] .
\end{aligned}
$$

Considerando que $k \in\{n, n+1, \cdots, p\}$, chega-se ao sistema de equações lineares em (12). 


\subsection{Extensão para o Caso Multivariável}

No caso de um sistema multivariável com $q$ entradas e $l$ saídas, considera-se que cada uma das saídas pode ser identificada independentemente, isto é, que o problema original pode ser modelado por um conjunto de $l$ problemas MISO com $q$ entradas independentes. Assim, para cada saída $i$, com $i \in\{1,2, \cdots, l\}$ :

$$
\mathcal{A}_{i}(\delta, \theta) y_{i, k}=\mathcal{B}_{1}(\delta, \theta) u_{1, k}+\cdots+\mathcal{B}_{q}(\delta, \theta) u_{q, k},
$$

em que, $y_{i, k}$ representa a saída $y_{i}$ no instante $t=k T$. Utilizando o Teorema 1 e desenvolvendo (13) de forma análoga ao caso SISO é possível determinar um sistema de equações lineares para calcular os coeficientes dependentes do parâmetro variante $\theta$ em $\mathcal{A}_{i}, \mathcal{B}_{1}, \cdots, \mathcal{B}_{q}$.

\section{2 Índices de Ajuste}

A avaliação do erro de ajuste entre o comportamento do modelo e do sistema dinâmico real é realizado em duas partes. Na primeira, denominada de erro de ajuste dos coeficientes, testa-se o erro no cálculo dos coeficientes durante o processo de identificação, a partir dos dados medidos na entrada e na saída do sistema físico. Utilizando (12) e $v=p-n+1$, define-se o vetor de erro de ajuste como:

$$
\begin{gathered}
E_{a}=B-A X \\
{\left[\begin{array}{c}
e_{a, 1} \\
e_{a, 2} \\
\vdots \\
e_{a, v}
\end{array}\right]=\left[\begin{array}{c}
b_{1}-a_{11} x_{1}-\cdots-a_{1 \nu} x_{\nu} \\
b_{2}-a_{21} x_{1}-\cdots-a_{2 \nu} x_{\nu} \\
\vdots \\
b_{v}-a_{v 1} x_{1}-\cdots-a_{v \nu} x_{\nu}
\end{array}\right],}
\end{gathered}
$$

e os índices de ajuste dos coeficientes como:

$$
\begin{gathered}
J_{2}^{a}=\left\|E_{a}\right\|_{2}=\sqrt{\left|e_{a, 1}\right|^{2}+\left|e_{a, 2}\right|^{2}+\cdots+\left|e_{a, v}\right|^{2}} \\
J_{\infty}^{a}=\left\|E_{a}\right\|_{\infty}=\max \left\{\left|e_{a, 1}\right|,\left|e_{a, 2}\right|, \cdots,\left|e_{a, v}\right|\right\} \\
\bar{J}_{2}^{a}=\frac{J_{2}^{a}}{v}
\end{gathered}
$$

Observa-se que $B$ é o vetor contendo as saídas medidas do sistema. Neste caso, avalia-se o erro na geração da saída atual, considerando que as saídas e entradas anteriores foram as medidas no sistema. Isto corresponde à simulação do modelo um passo a frente.

Na segunda parte, com o modelo já identificado, avaliase o erro deste para uma dada simulação, o que é bem mais rigoroso. Adota-se um novo sinal de entrada para a validação e as mesmas condições iniciais para o sistema e para o modelo. Neste caso, considera-se a simualção livre do modelo, isto é, a sua saída $\hat{y}_{k}$ é gerada exclusivamente a partir da entrada fornecida e do histórico do parâmetro variável $\theta$, sem a interferência da saída $y$ do sistema, conforme mostra a figura 1.

O erro para um horizonte de $h$ períodos de simulação é avaliado da seguinte forma:

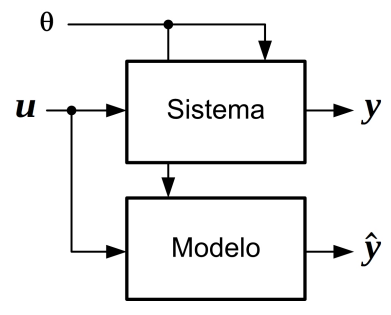

Figura 1. Simulação livre do modelo a partir da entrada e do parâmetro variante $\theta$.

$$
E_{s}=\left[\begin{array}{c}
e_{s, 1} \\
e_{s, 2} \\
\vdots \\
e_{s, h}
\end{array}\right]=\left[\begin{array}{c}
y_{1}-\hat{y}_{1} \\
y_{2}-\hat{y}_{2} \\
\vdots \\
\vdots \\
y_{h}-\hat{y}_{h}
\end{array}\right]=\boldsymbol{y}-\hat{\boldsymbol{y}}
$$

e os índices de simulação ou validação por:

$$
\begin{gathered}
J_{2}^{s}=\left\|E_{s}\right\|_{2}=\sqrt{\left|e_{s, 1}\right|^{2}+\left|e_{s, 2}\right|^{2}+\cdots+\left|e_{s, h}\right|^{2}} \\
J_{\infty}^{s}=\left\|E_{s}\right\|_{\infty}=\max \left\{\left|e_{a, 1}\right|,\left|e_{s, 2}\right|, \cdots,\left|e_{s, h}\right|\right\} \\
\bar{J}_{2}^{s}=\frac{J_{2}^{s}}{h}
\end{gathered}
$$

Cabe observar que se $v=h$, então $J_{2}^{a} \leq J_{2}^{s}$.

\section{EXEMPLOS DE APLICAÇÃO}

Nesta seção, encontram-se apresentados dois exemplos. O primeiro se refere a um sistema Quasi-LPV do tipo massamola com dois estados, em que o parâmetro variante é também um dos estados. O segundo é um exemplo de quarta ordem, em que dois de seus coeficientes dependem de funções senoidais do parâmetro variante $\theta$.

\subsection{Sistema Massa-Mola-Amortecedor com 2 Estados}

A figura 2 ilustra um sistema massa-mola-amortecedor de $2^{a}$ ordem, apoiado em uma superfície plana. A entrada deste sistema é a força $F$ aplicada ao bloco de massa $m_{a}$ e a saída a posição $y$ do bloco. A constante $b$ representa o coeficiente de atrito viscoso e $K$ é a constante elástica da mola.

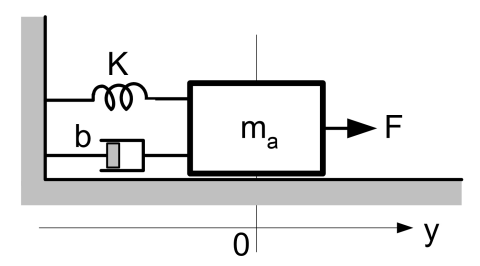

Figura 2. Sistema massa-mola-amortecedor de $2^{a}$ ordem.

Desprezando o atrito na superfície e aplicando a segunda lei de Newton:

$$
F-F_{K}-F_{b}=m_{a} \ddot{y}
$$

em que $F_{K}=K y$ e $F_{b}=b \dot{y}$. Assim, a equação diferencial ordinária que representa a dinâmica é dada por: 


$$
\ddot{y}+\frac{b}{m_{a}} \dot{y}+\frac{K}{m_{a}} y=\frac{F}{m_{a}} .
$$

As varáveis de estado podem ser arbitradas como: $x_{1}=y$ a posição da massa; e $x_{2}=\dot{y}$ a velocidade da massa. Uma representação em espaço de estado desse sistema seria dada por:

$$
\begin{gathered}
{\left[\begin{array}{c}
\dot{x}_{1} \\
\dot{x}_{2}
\end{array}\right]=\left[\begin{array}{cc}
0 & 1 \\
-\frac{K}{m_{a}} & -\frac{b}{m_{a}}
\end{array}\right]\left[\begin{array}{l}
x_{1} \\
x_{2}
\end{array}\right]+\left[\begin{array}{c}
0 \\
\frac{1}{m_{a}}
\end{array}\right] F,} \\
y=\left[\begin{array}{ll}
1 & 0
\end{array}\right]\left[\begin{array}{l}
x_{1} \\
x_{2}
\end{array}\right]
\end{gathered}
$$

A fim de discretizar (20) e transformá-la em uma série temporal, no formato de (7), considere:

$$
\begin{aligned}
& \dot{y}(k T) \triangleq \lim _{T \rightarrow 0} \frac{y((k+1) T)-y(k T)}{T} \approx \frac{y_{k+1}-y_{k}}{T}, \\
& \ddot{y}(k T) \approx \frac{\dot{y}_{k+1}-\dot{y}_{k}}{T} \approx \frac{y_{k+2}-2 y_{k+1}+y_{k}}{T^{2}} .
\end{aligned}
$$

Substituindo em (20), chega-se a:

$$
y_{k+2}=\left(2-\frac{b T}{m_{a}}\right) y_{k+1}+\left(\frac{b T}{m_{a}}-\frac{K T^{2}}{m_{a}}-1\right) y_{k}+\frac{T^{2}}{m_{a}} F_{k} .
$$

Com o objetivo de inserir uma não-linearidade no modelo, a constante elástica da mola foi considerada variável e dependente da posição da massa, ou seja, $K=\cos (\theta)$, onde $\theta=y$. Além disso, a título de simulação, adotou-se $m_{a}=1 \mathrm{~kg}, b=0,1 \mathrm{Ns} / \mathrm{m}$ e $T=0,01 \mathrm{~s}$. As figuras $3 \mathrm{e}$ 4 apresentam, respectivamente, os dados de entrada e de saída utilizados na identificação e validação dos modelos.
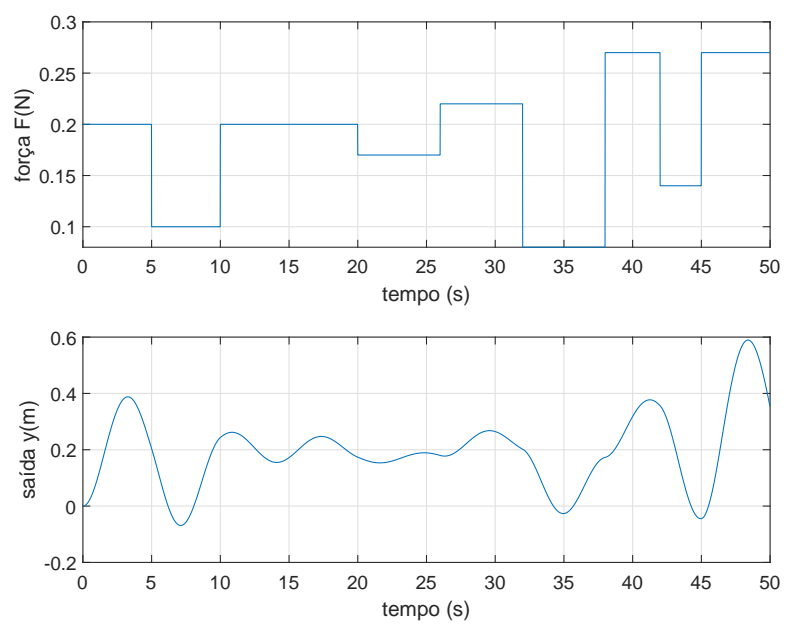

Figura 3. Dados de entrada e saída para a identificação.

A tabela 1 apresenta as características dos três modelos identificados para o sistema massa-mola. O modelo Ex1-1 é uma aproximação LTI. O modelo Ex1-2, cujos coeficientes ajustados são apresentados no Apêndice A, é o que apresentou o melhor ajuste para o sistema massa-mola e, pelo princípio da parcimônia, seria o modelo escolhido para
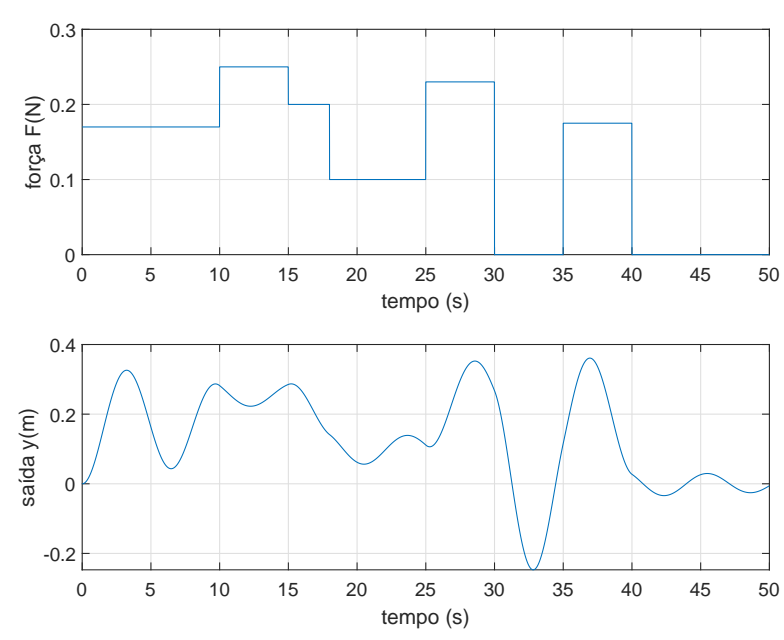

Figura 4. Dados de entrada e saída para a validação.

uma representação LPV polinomial do sistema em análise. O modelo Ex1-3 foi uma tentativa de identificação com ordem superior. Embora seu ajuste tenha sido bom, não foi melhor que o do Ex1-2.

Tabela 1. Características dos modelos identificados para o sistema massa-mola.

\begin{tabular}{rrrr} 
Modelo & Ex1-1 & Ex1-2 & Ex1-3 \\
\hline$n$ & 2 & 2 & 3 \\
$m$ & 1 & 1 & 1 \\
$r$ & 2 & 2 & 2 \\
$c$ & 1 & 1 & 1 \\
$\mathbf{N}$ & {$[00]$} & {$[22]$} & {$[222]$} \\
$\mathbf{M}$ & {$[0]$} & {$[1]$} & {$[1]$} \\
$\nu$ & 3 & 8 & 11 \\
$J_{2}^{a}$ & $7,5028 \mathrm{e}-5$ & $2,4636 \mathrm{e}-7$ & $5,0189 \mathrm{e}-3$ \\
$J_{\infty}^{a}$ & $4,9208 \mathrm{e}-6$ & $1,3043 \mathrm{e}-8$ & $1,7627 \mathrm{e}-4$ \\
$\overline{J_{2}^{a}}$ & $1,5012 \mathrm{e}-8$ & $4,9291 \mathrm{e}-11$ & $1,0044 \mathrm{e}-6$ \\
$v$ & 4998 & 4998 & 4997 \\
$J_{2}^{s}$ & $1,4020 \mathrm{e}+0$ & $3,9115 \mathrm{e}-2$ & $6,0013 \mathrm{e}-2$ \\
$J_{\infty}^{s}$ & $4,9393 \mathrm{e}-2$ & $1,7712 \mathrm{e}-3$ & $2,0364 \mathrm{e}-3$ \\
$\overline{J_{2}^{s}}$ & $2,8052 \mathrm{e}-4$ & $7,8261 \mathrm{e}-6$ & $1,2010 \mathrm{e}-5$ \\
$h$ & 4998 & 4998 & 4997 \\
$\max |\lambda|$ & 0,9995 & 0,9996 & 0,9996 \\
\hline
\end{tabular}

A figura 5 ilustra a saída do modelo LTI Ex1-1, em vermelho, e a saída do sistema massa-mola, em azul.

A figura 6 ilustra: (a) o erro entre a saída do modelo Ex1-2 e do sistema massa-mola; e (b) o máximo módulo dos polos do modelo LPV Ex1-2, para valores fixos do parâmetro, ao longo da validação. Vale observar que o sinal de saída do modelo Ex1-2 coincide com a saída do sistema massa-mola, em azul, na figura 5, o que é corroborado com os valores apresentados por seus índices $J_{2}^{s}$ e $J_{\infty}^{s}$ na tabela 1 .

\subsection{Exemplo com 4 Estados}

Neste exemplo, ilustra-se as possibilidades da metodologia proposta na identificação do modelo de um sistema LPV de quarta ordem, em que alguns coeficientes são funções não-lineares do parâmetro variante $\theta$. O modelo utilizado para o sistema a ser identificado é: 


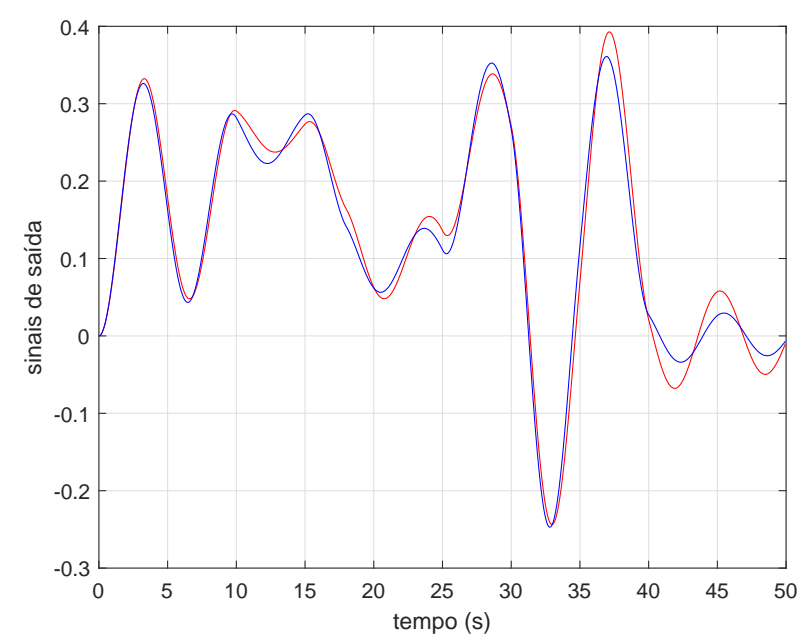

Figura 5. Saídas do modelo Ex1-1 (verm.), em simulação livre, e do sistema (azul) para a entrada de validação.
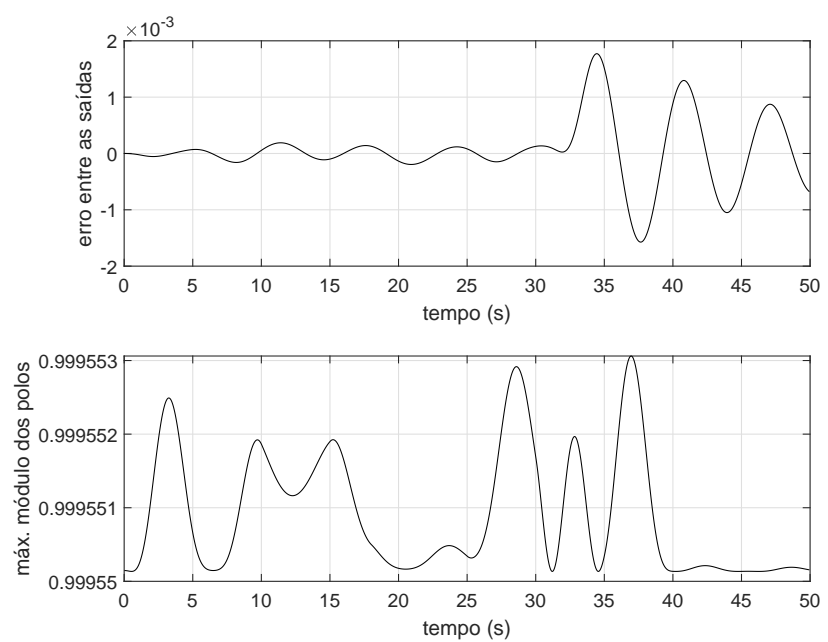

Figura 6. (a) Erro entre as saídas do modelo Ex1-2 e do sistema. (b) Valor máximo de módulo dos polos do modelo Ex1-2.

$$
\begin{array}{r}
y_{k}=\left[2,475+0,025 \cos \left(\theta_{k-1}\right)\right] y_{k-1}-2,36 y_{k-2}+ \\
+\left[1-0,01 \operatorname{sen}\left(\theta_{k-1}\right)\right] y_{k-3}-0,16 y_{k-4}+ \\
+u_{k-3}-0,1 u_{k-4} .
\end{array}
$$

As figuras 7 e 8 apresentam, respectivamente, os dados de entrada e de saída utilizados na identificação e validação dos modelos. Para efeito de simulação, considerou-se que o modelo acima tenha sido discretizado $\operatorname{com} T=0,01 \mathrm{~s}$ e que o parâmetro variante $\theta$ esteja excursionando no intervalo $[0,1]$. Mais precisamente, considerou-se que nos $10 \mathrm{~s}$ dos dados de entrada para a identificação, na figura $7, \theta$ variou linearmente de 1 até 0 .

A tabela 2 apresenta as características dos quatro modelos identificados para o sistema proposto no exemplo de quarta ordem. Os modelos Ex2-1, Ex2-2 e Ex2-3 representam uma identificação paramétrica com a redução progressiva do erro de ajuste, mas às custas do aumento nas ordens dos polinômios nos coeficientes do modelo. O modelo Ex2-1 é uma aproximação LTI do sistema LPV, pois os elementos
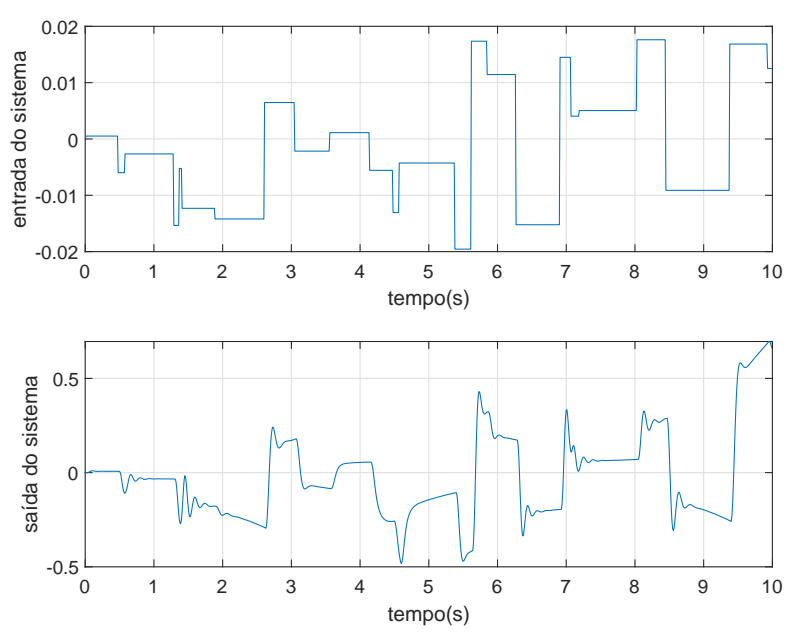

Figura 7. Dados de entrada e saída para a identificação.
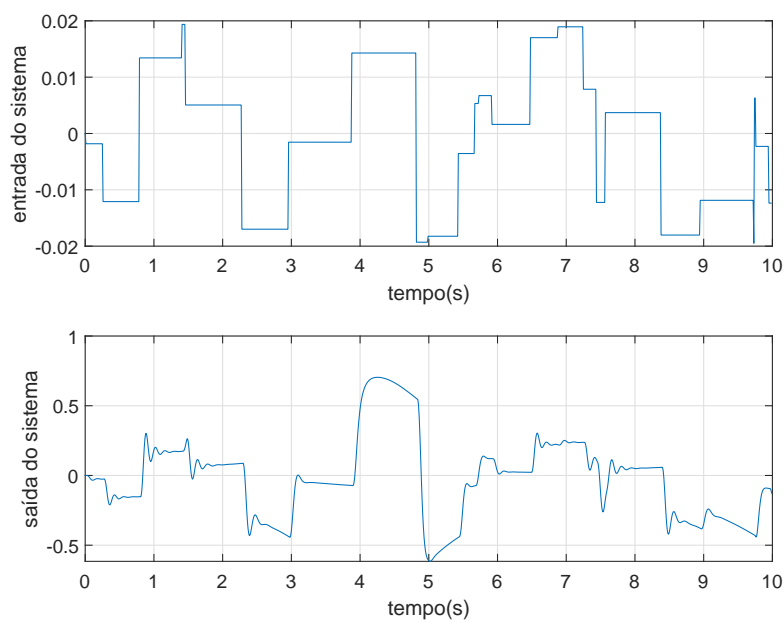

Figura 8. Dados de entrada e saída para a validação.

de $\mathbf{N}$ e $\mathbf{M}$ foram zerados para o cálculo do modelo, conforme mostra a tabela 2. O modelo Ex2-4 ilustra uma identificação com ordem reduzida.

\begin{tabular}{|c|c|c|c|c|}
\hline Modelo & Ex2-1 & Ex2-2 & Ex2-3 & Ex2-4 \\
\hline$n$ & 4 & 4 & 4 & 2 \\
\hline$m$ & 2 & 2 & 2 & 1 \\
\hline$r$ & 3 & 3 & 3 & 2 \\
\hline$c$ & 1 & 1 & 1 & 1 \\
\hline $\mathbf{N}$ & {$\left[\begin{array}{llll}0 & 0 & 0 & 0\end{array}\right]$} & {$\left[\begin{array}{llll}3 & 1 & 3 & 1\end{array}\right]$} & {$\left[\begin{array}{llll}5 & 2 & 5 & 2\end{array}\right]$} & {$\left[\begin{array}{ll}5 & 5\end{array}\right]$} \\
\hline $\mathbf{M}$ & {$\left[\begin{array}{ll}0 & 0\end{array}\right]$} & {$\left[\begin{array}{ll}2 & 2\end{array}\right]$} & {$\left[\begin{array}{ll}2 & 2\end{array}\right]$} & [3] \\
\hline$\nu$ & 6 & 18 & 24 & 16 \\
\hline$J_{2}^{a}$ & $1,3357 \mathrm{e}-2$ & $9,1343 \mathrm{e}-3$ & $2,1125 \mathrm{e}-3$ & $1,3663 \mathrm{e}-1$ \\
\hline$J_{\infty}^{a}$ & $3,4336 \mathrm{e}-3$ & $2,3684 \mathrm{e}-3$ & $4,7200 \mathrm{e}-4$ & $4,4233 \mathrm{e}-2$ \\
\hline $\bar{J}_{2}^{a}$ & $1,3357 \mathrm{e}-5$ & $9,1343 \mathrm{e}-6$ & $2,1125 \mathrm{e}-6$ & $1,3636 \mathrm{e}-4$ \\
\hline$v$ & 1000 & 1000 & 1000 & 1002 \\
\hline$J_{2}^{s}$ & $4,6252 \mathrm{e}+0$ & $2,4534 \mathrm{e}+0$ & $1,1185 \mathrm{e}-1$ & $1,0987 \mathrm{e}+0$ \\
\hline$J_{\infty}^{s}$ & 4,1941e-1 & $2,5830 \mathrm{e}-1$ & $1,5916 \mathrm{e}-2$ & $1,7079 \mathrm{e}-1$ \\
\hline$\overline{J_{2}^{s}}$ & $4,6252 \mathrm{e}-3$ & $2,4534 \mathrm{e}-3$ & $1,1185 \mathrm{e}-4$ & $1,0965 \mathrm{e}-3$ \\
\hline$h$ & 1000 & 1000 & 1000 & 1002 \\
\hline $\max |\lambda|$ & 0,9833 & 1,0432 & 0,9111 & 0,9452 \\
\hline
\end{tabular}

Tabela 2. Características dos modelos obtidos para o exemplo de quarta ordem em (22). 
A figura 9 apresenta as saídas do modelo identificado LTI Ex2-1 e do sistema LPV em (22).

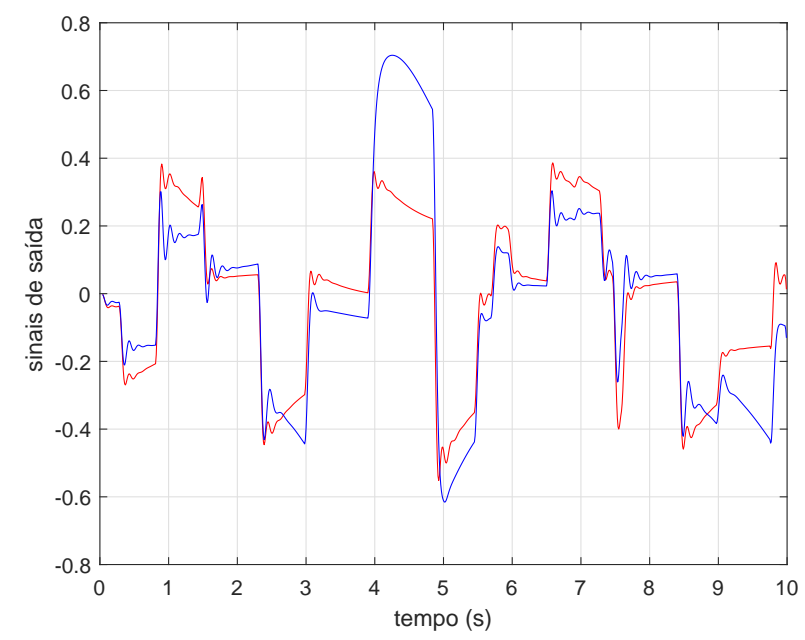

Figura 9. Saídas do modelo Ex2-1 (verm.), em simulação livre, e do sistema (azul) para a entrada de validação.

A figura 10 se refere ao modelo LPV Ex2-3 que obteve o melhor ajuste para o sistema em (22), com os coeficientes apresentados no Apêndice A, e apresenta: (a) o erro entre as saídas desse modelo e do sistema; e (b) o máximo módulo dos polos ao longo da validação. Devido ao pequeno erro, a saída desse modelo para a entrada de validação equivale a curva em azul na figura 9. Vale observar que o máximo módulo dos polos encontra-se sempre com valor inferior a 1, que representa a estabilidade do modelo invariante no domínio discreto ao longo da trajetória do parâmetro. Esse gráfico se mostrou uma importante ferramenta de análise da qualidade do modelo LPV durante a fase de identificação. Verificou-se também, em alguns casos, que pequenas variações acima do valor unitário pelo módulo máximo dos polos, por poucos períodos $T$, nem sempre ocasionam instabilidade na simulação do modelo.
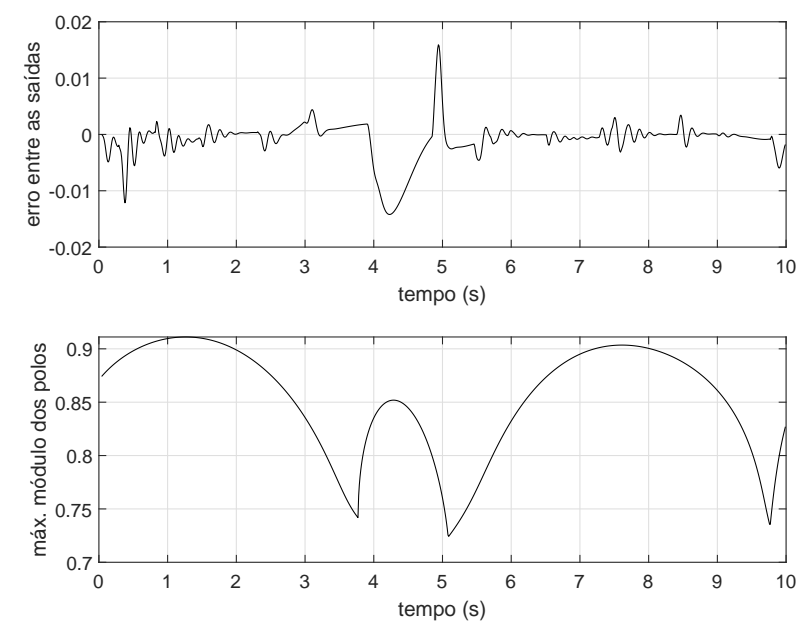

Figura 10. (a) Erro entre o modelo identificado Ex2-3 e o sistema em (22). (b) Valor máximo de módulo dos polos do modelo Ex2-3.
As figuras 11 e 12 se referem ao modelo Ex2-4, que apresenta uma estrutura reduzida em relação ao modelo LPV em (22) a ser identificado. Analisando a tabela 2, verifica-se pelos índices de validação, $J_{2}^{s}$ e $J_{\infty}^{s}$, que o desempenho do modelo Ex2-4 foi melhor que os dos Ex2-1 e Ex2-2, sendo que em relação a este último, a diminuição do número de parâmetros do modelo foi compensada pelo aumento das ordens dos polinômios nos coeficientes de Ex2-4.

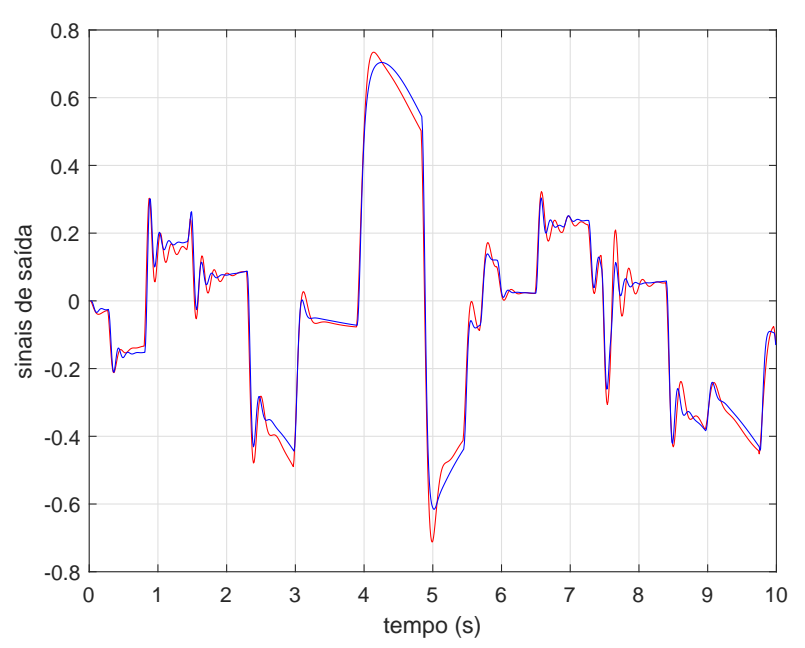

Figura 11. Saídas do modelo Ex2-4 (verm.), em simulação livre, e do sistema (azul) para a entrada de validação.
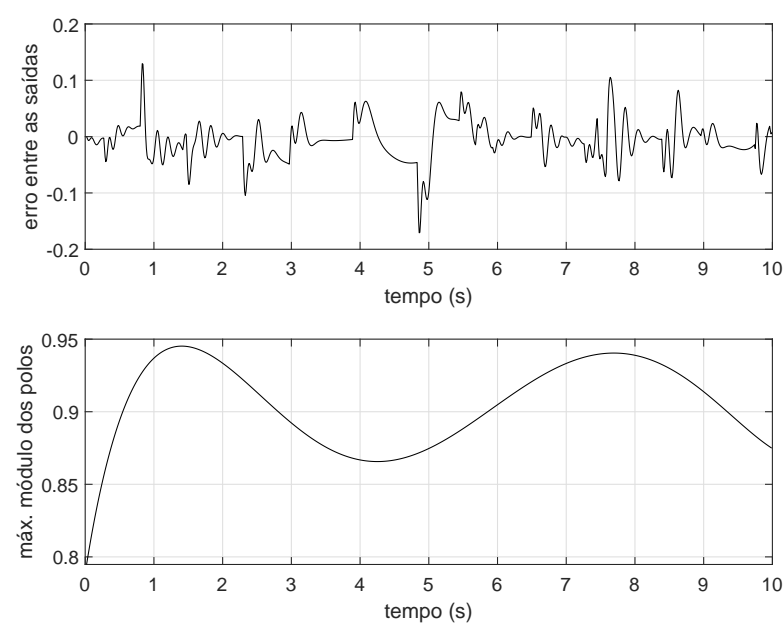

Figura 12. (a) Erro entre o modelo identificado Ex2-4 e o sistema em (22). (b) Valor máximo de módulo dos polos do modelo Ex2-3.

As figuras 13 e 14 se referem ao modelo Ex2-3r, que foi identificado a partir de dados contaminados com ruído branco e média nula, na proporção de até $\pm 2 \%$ nos dados de saída do sistema. Esse modelo possui a mesma estrutura do Ex2-3, na tabela 2, e apresenta os seguintes índices de ajuste: $J_{2}^{s}=5,3502 e-1$ e $J_{\infty}^{s}=7,9626 e-2$. Comparando as figuras 10 e 14 verifica-se que o erro entre as saídas do modelo identificado e o sistema aumentou 5 vezes com a introdução do ruído. Apesar desse aumento, a figura 13 ilustra que o modelo Ex2-3r também obteve um bom ajuste. 


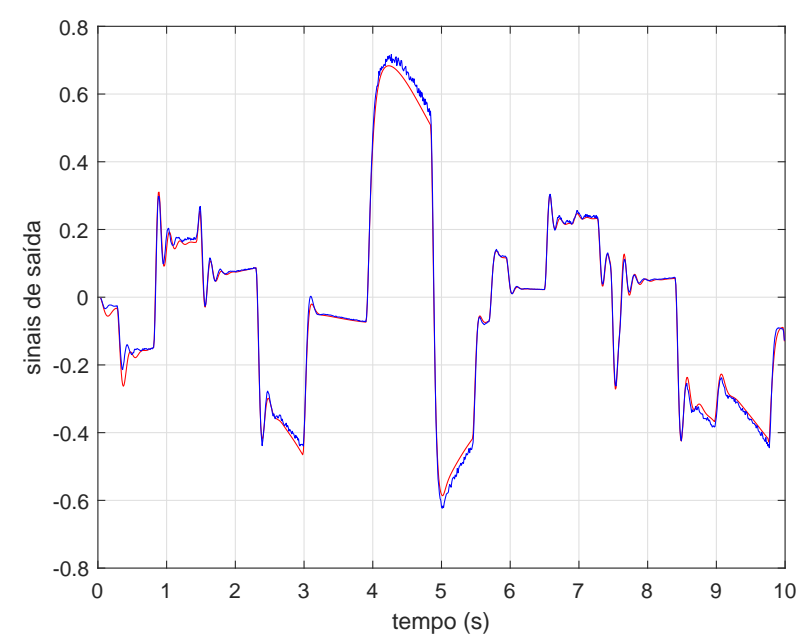

Figura 13. Saídas do modelo identificado com ruído Ex2-3r (verm.), em simulação livre, e do sistema (azul) para a entrada de validação.
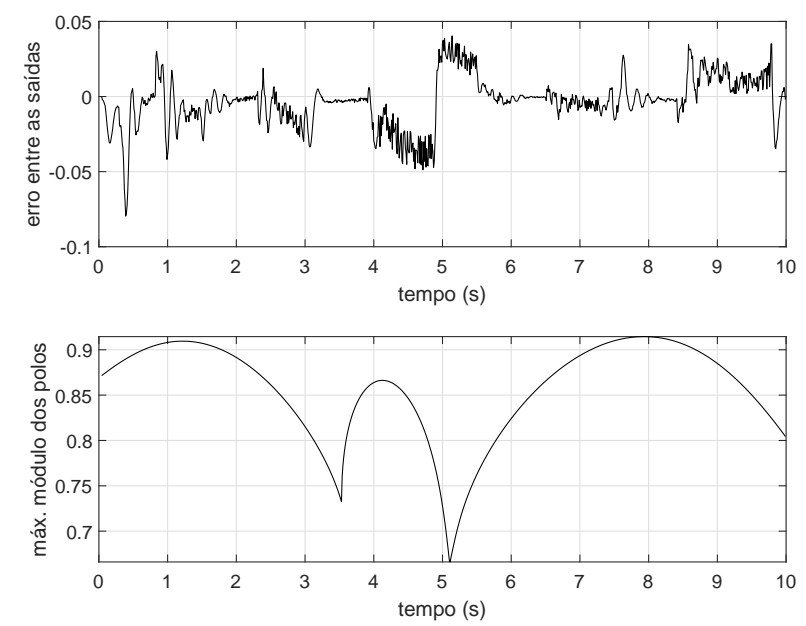

Figura 14. (a) Erro entre o modelo identificado com ruído Ex2-3r e o sistema em (22). (b) Valor máximo de módulo dos polos do modelo Ex2-3.

\section{CONCLUSÕES}

Neste artigo, apresentou-se um método multivariável para a identificação de modelos LPV a fim de aproximar sistemas com comportamento possivelmente não-linear. Os resultados alcançados nos exemplos foram satisfatórios. Algumas contribuições em relação as ideias de (Bamieh and Giarré, 1999, 2002) foram implementadas, como a extensão do método para sistemas multivariáveis, a obtenção da solução a partir de um lote de dados, a utilização de polinômios com graus independentes por parâmetro, e a possibilidade de realizar a defasagem temporal entre a saída corrente e a entrada mais recente no modelo. Em termos de esforço computacional, o problema não se mostrou significativo caso o modelo tenha sua estrutura previamente arbitrada. Além disso, observou-se que o gráfico que mostra o máximo módulo dos polos do modelo para parâmetros fixos ao longo da simulação foi uma im- portante ferramenta para analisar a qualidade do modelo identificado.

\section{REFERÊNCIAS}

Aguirre, L.A. (2015). Introdução à Identificação de Sistemas. Técnicas Lineares e Não Lineares: Teoria e Aplição. Editora UFMG.

Bamieh, B. and Giarré, L. (1999). Identification of linear parameter varying models. In Proceedings of the 38th IEEE conference on decision and control, Phoenix, AZ, December.

Bamieh, B. and Giarré, L. (2002). Identification of linear parameter varying models. Journal of Robust and Nonlinear Control 12, 841-853.

Becker, G.S. (1993). Quadratic stability and performance of linear parameter dependent systems. Ph.D. thesis, Department of Mehanial Engineering, University of California-Berkeley, USA.

Ljung, L. (1999). System Identification: Theory for the User Second Edition. Prentice Hall PTR.

Ljung, L. and Glad, T. (2016). Modeling and identification of dynamic system. Studentlitteratur.

Lovera, M., Bergamasco, M., and Casella, F. (2013). Robust Control and Linear Parameter Varying Approaches, volume 437 of Lecture Notes in Control and Information Sciences, chapter LPV Modelling and Identification: An Overview, 3-24. Springer, Berlin, Heidelberg.

Marcos, A. and Balas, G.J. (2004). Development of linear-parameter-varying models for aircraft. Journal of Guidance, Control, and Dynamics, 27(2), 218-228.

Rugh, W.J. and Shamma, J.S. (2000). Survey paper research on gain scheduling. Automatica 36, pp 1401 $-1425$.

Verdult, V. (2002). Nonlinear System Identification: A State-Space Approach. Ph.D. thesis, Univ. of Twente.

\section{Apêndice A. COEFICIENTES DE MODELOS}

Tabela A.1. Coeficientes dos modelos Ex1-2 e Ex2-3 identificados.

\begin{tabular}{rrr} 
Ex1-2 & Ex2-3 & Ex2-3 \\
\hline $\mathrm{X}$ & $X_{\alpha}$ & $X_{\beta}$ \\
\hline$-1,9990 \mathrm{e}+0$ & $-2,2444 \mathrm{e}+0$ & $9,9960 \mathrm{e}-1$ \\
$9,7578 \mathrm{e}-7$ & $-2,3435 \mathrm{e}-1$ & $6,3181 \mathrm{e}-4$ \\
$-9,7648 \mathrm{e}-5$ & $6,4417 \mathrm{e}-2$ & $-1,7033 \mathrm{e}-4$ \\
$9,9910 \mathrm{e}-1$ & $-1,3838 \mathrm{e}-2$ & $1,7358 \mathrm{e}-1$ \\
$-2,1821 \mathrm{e}-6$ & $1,7602 \mathrm{e}-3$ & $-2,4406 \mathrm{e}-1$ \\
$5,0765 \mathrm{e}-5$ & $-7,4271 \mathrm{e}-5$ & $2,9238 \mathrm{e}-2$ \\
$9,9978 \mathrm{e}-5$ & $1,7914 \mathrm{e}+0$ & \\
$1,0254 \mathrm{e}-7$ & $4,8085 \mathrm{e}-1$ & \\
& $-5,6960 \mathrm{e}-2$ & \\
& $-5,5765 \mathrm{e}-1$ & \\
& $-3,4291 \mathrm{e}-1$ & \\
& $2,2629 \mathrm{e}-2$ & \\
& $4,7901 \mathrm{e}-3$ & \\
& $-4,2055 \mathrm{e}-4$ & \\
& $1,2847 \mathrm{e}-5$ & \\
& $3,6919 \mathrm{e}-2$ & \\
& $1,0241 \mathrm{e}-1$ & \\
\hline
\end{tabular}

